\title{
The PerPot Simulated Anaerobic Threshold - A Comparison to Typical Lactate-based Thresholds
}

\author{
Stefan Endler ${ }^{*}$, Sabine Hoffmann, Björn Sterzing, Perikles Simon, Mark Pfeiffer \\ Institute of Sport Science, Johannes Gutenberg-University, Germany
}

Copyright $\bigcirc 2017$ by authors, all rights reserved. Authors agree that this article remains permanently open access under the terms of the Creative Commons Attribution License 4.0 International License

\begin{abstract}
Intensity description as exercise prescription is a main challenge for sport scientists and coaches. Most commonly used in endurance sports are percentages of the heart rate at Maximal Lactate Steady State (MLSS). Since the late $1970 \mathrm{~s}$, MLSS is approximated by several anaerobic thresholds based on lactate extraction during graded incremental tests. Since then, scientists look for non-invasive methods to approximate these thresholds. Our new approach uses the sports computer science Performance Potential model (PerPot) for determination. The antagonistic model PerPot models the relation between load (speed) and performance (heart rate). This investigation compares lactate based and PerPot simulated thresholds. Fifteen male handball players performed a graded incremental test with lactate extraction and continuous heart rate recording. Lactate measurement was used for determination of four established lactate-based anaerobic thresholds (OBLA, Dickhuth, Keul, Simon). Speed and heart rate processes were used for PerPot determination of the threshold. Both Pearson correlations $(\mathrm{r}=0.883-\mathrm{r}=0.895)$ and intraclass correlations $(\mathrm{ICC}=0.894-\mathrm{ICC}=0.932)$ show high correlations between lactate-based and PerPot simulated thresholds. Using anaerobic threshold (AT) for exercise prescription is the ideal case. Because of adaptation, AT changes and should therefore be determined periodically. In practice, this is rarely done because of high cost and difficult invasive determination in laboratory. PerPot provides a low cost, non-invasive method for AT determination. It is therefore an ideal method for verifying former results of sports medicine diagnostics periodically. In addition, simple portability of results to field is an advantage, because the graded incremental tests can also be performed outdoors.
\end{abstract}

Keywords Physical Endurance, Exercise, Modeling, Performance

\section{Introduction}


incremental tests [11]. In 2009, a sports computer science method was developed using multi-layer perceptrons for estimating the fixed AT at $4 \mathrm{mmol} / \mathrm{l}$ [12]. These neural networks were generated with anthropometric data and HR of the last five stages of a graded incremental test of 113 soccer players. The disadvantage of this approach is the model itself. It estimates the AT by searching for general patterns using the underlying data. ATs can only be estimated for athletes with similar anthropometry, because of no correspondence between the model and the individuality of physiology. In contrast, our Performance Potential model (PerPot) reproduces the individual behaviour of HR on speed. ATs can be identified by simulating several constant speed endurance runs. The aim of the present investigation was to compare our simulated AT with the most common lactate-based ATs in practice. For this purpose, fifteen well-trained male handball players performed a graded incremental test.

\section{Materials and Methods}

\subsection{Subjects}

Fifteen male handball players were recruited for this study but only 13 (age: $23.2 \pm 2.3$; height (cm): 186.2 \pm 7.8 ; weight (kg): $88.3 \pm 11.4$ ) were included for data analysis at the end of the study. The G*Power analyses [13] reveals a minimum of 13 participants to show significance in very high correlations $(r>0.9)$. One participant was excluded because of incorrect data measurement and the other one was excluded because the graded incremental test had to be interrupted before the participant reached his subjective exhaustion due to blood pressure problems. Table 1 shows their age and physical characteristics, which were measured before our tests.

Table 1. Anthropometric data and training characteristics of the subjects $(\mathrm{N}=13)$

\begin{tabular}{|c|c|c|c|}
\hline & Min & Max & Mean $\pm \mathrm{s}$ \\
\hline Age (Years) & 20 & 27 & $23,23 \pm 2,28$ \\
\hline Body mass $(\mathrm{kg})$ & 70,9 & 110,6 & $88,30 \pm 11,41$ \\
\hline Height $(\mathrm{cm})$ & 174,0 & 200,0 & $186,15 \pm 7,82$ \\
\hline BMI $\left(\mathrm{kg} / \mathrm{m}^{2}\right)$ & 23,2 & 29,7 & $25,40 \pm 2,03$ \\
\hline $\begin{array}{c}\mathrm{VO}_{2} \mathrm{max}(\mathrm{ml} \\
\left.\mathrm{O}_{2} / \min \right)\end{array}$ & 44,7 & 54,9 & $50,72 \pm 3,27$ \\
\hline
\end{tabular}

The present study was approved by the Ethics Committee of the Johannes Gutenberg University in Mainz. All participants had a minimum load of three exercises or two exercises and a competition per week. Furthermore, they were all non-smokers and had no cardiological illness. Intensive or long continuous exercise and alcoholic consumption was forbidden one day before the graded incremental test.
The graded incremental test was executed on a treadmill adjusted with a gradient of $1.5 \%$. All tests started with an initial workload of $6 \mathrm{~km} / \mathrm{h}$ for 3 minutes and incremental 2 $\mathrm{km} / \mathrm{h}$ increases every 3 minutes until subjective exhaustion was reached with a minimum of five steps. Between steps, a 30 second break took place for capillary blood extraction. Subsequent to the last incremental step, another step of 3 minutes with the previous initial workload of $6 \mathrm{~km} / \mathrm{h}$ took place.

All tests were performed for standardization between 1 and $7 \mathrm{PM}$ to minimize any possible influence of circadian cycle.

The capillary blood sample extractions were carried out before the beginning of graded incremental test, in each 30 second break between steps and after the 1st, 3rd, 5th and 20th minute subsequent to subjective exhaustion break off. To determine the lactate concentration blood samples were extracted using a $20 \mu \mathrm{l}$ sodium- heparinized end-to-end plastic capillary from ear lobe, which was hyperemic-based with Finalgon ${ }^{\circledR}$ and was cleaned with disinfectant. Samples were stored at $5-8^{\circ} \mathrm{C}$ temperature until analysis. Lactate concentrations were determined with the measurement device BIOSEN 5140 ® by EFK electrochemically. These lactate concentrations and all heart rate values at the end of particular corresponding step were used as input for the analysis software Winlactat 3.3 by Mesics GmbH. Thereby, heart rate values at AT were determined with different mathematical models. In this study, we decided to determine the most common and well-established thresholds " +1.5 $\mathrm{mmol} / \mathrm{l}$ " by Dickhuth [14], onset of blood lactate accumulation (OBLA) by Mader [15], Keul [16] and Simon [17]. Instead of the original $4 \mathrm{mmol} / \mathrm{l}$ OBLA, we decided to take a lower onset of $3.5 \mathrm{mmol} / \mathrm{l}$ because of the shorter step duration of 3 minutes compared to the originally used 5 minutes step duration [18].

In contrast to standard lactate based sport medical performance diagnostic, where only one heart rate value at the end of a step is taken, we needed a continuous heart rate profile for PerPot usage. Thus, a heart rate recording device was applied during graded incremental tests, which recorded heart rates in five second intervals. A Polar ${ }^{\circledR}$ heart rate monitor by Polar ${ }^{\circledR}$ Electro Oy, consisted of electrode belt and transmitter WearLink ${ }^{\circledR}$ W.I.N.D. and heart rate monitor RS800CX was used for this purpose. Data was transmitted to a computer via an infrared interface and was visualized and edited using the Polar ProTrainer $5^{\mathrm{TM}}$ software. After transmission, the data was available as .hrm file for further calculations [19].

The corresponding speed was determined exactly from the treadmill, including acceleration and breaking. Speed values were determined in 5 second intervals similar to heart rate measurement and finally included into the .hrm file, which was used as data input for the PerPot analysis. The simulated IAT heart rate values were determined with the PerPot web version on www.perpot.de as of March 2017. 
The goal of the model was the reproduction of a HR progress by a given speed progress. Particularly, the delayed adaptation of HR on a changing speed should be reproduced by the model. This delayed (asymptotic) behaviour of HR can be reproduced by PerPot, which was adapted among other things for running [20].

In general, PerPot is a discrete and deterministic computer science model to represent interdependencies between load input and performance output [21]. In our scenario, load input and performance output correspond to running speed and HR, respectively. The effect of running speed on heart rate results from two potentials. The strain potential (SP) increments HR, whereas the recovery potential (RP) decrements HR. Both effects occur with different time delays, i.e. the strain delay (DS) and the recovery delay (DR).

PerPot has a further mechanism to react on overloading. If the running speed is getting too high, SP reaches its maximum and the overload mechanism provoke a shorter delay of the negative effect to HR in order to avoid an endless increase of HR (see figure 1).

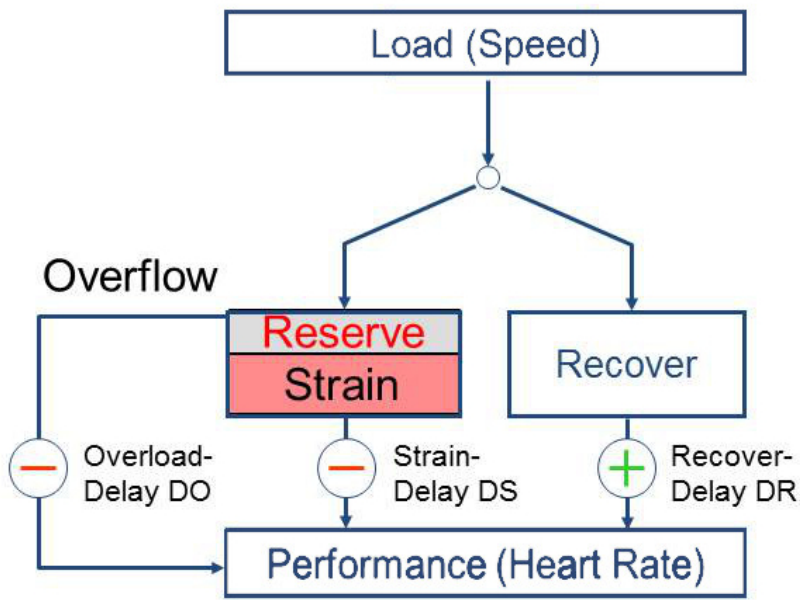

Figure 1. Structure of the performance potential model (PerPot)

The difference equations of the interim values SP and RP and the output value HR are as follows:

$$
\begin{gathered}
\quad \operatorname{SP}[\mathrm{t}+\Delta \mathrm{t}]=\operatorname{SP}[\mathrm{t}]+\Delta \mathrm{t} \cdot(\operatorname{Speed}[\mathrm{t}]- \\
-\operatorname{SR}[\mathrm{t}, \mathrm{t}+\Delta \mathrm{t}]-\mathrm{OR}[\mathrm{t}, \mathrm{t}+\Delta \mathrm{t}]) \\
\mathrm{RP}[\mathrm{t}+\Delta \mathrm{t}]=\mathrm{RP}[\mathrm{t}]+\Delta \mathrm{t} \cdot(\operatorname{Speed}[\mathrm{t}]- \\
-\operatorname{RR}[\mathrm{t}, \mathrm{t}+\Delta \mathrm{t}]) \\
\operatorname{HR}[\mathrm{t}+\Delta \mathrm{t}]=\operatorname{HR}[\mathrm{t}]+\Delta \mathrm{t}(\operatorname{RR}[\mathrm{t}, \mathrm{t}+\Delta \mathrm{t}]- \\
-\operatorname{SR}[\mathrm{t}, \mathrm{t}+\Delta \mathrm{t}]-\text { OR }[\mathrm{t}, \mathrm{t}+\Delta \mathrm{t}])
\end{gathered}
$$

The three rates i.e. strain rate (SR), recover rate (RR) and overflow rate (OR) are determined by the following equations:

$$
\begin{array}{r}
\mathrm{SR}[\mathrm{t}, \mathrm{t}+\Delta \mathrm{t}]=\min (1, \mathrm{SP}[\mathrm{t}], \mathrm{PP}[\mathrm{t}]) / \mathrm{DS} \\
\mathrm{RR}[\mathrm{t}, \mathrm{t}+\Delta \mathrm{t}]=\min (1, \mathrm{RP}[\mathrm{t}], 1-\mathrm{PP}[\mathrm{t}]) / \mathrm{DR}
\end{array}
$$

$$
\mathrm{OR}[\mathrm{t}, \mathrm{t}+\Delta \mathrm{t}]=\max (0, \mathrm{SP}[\mathrm{t}]-1) / \mathrm{DO}
$$

As one can see, the rates could also result from PP. This possible back coupling causes an S-shaped HR response given an incremental speed process [22].

\subsection{Calibration and Simulation}

For AT determination using the model, two steps are needed, the individual calibration step and the simulation step.

Since variation of running speeds causes different delayed HR reaction in individuals, the model delays have to be calibrated for every athlete, individually. Therefore, the graded incremental test described above was conducted to determine the individual delayed changes of heart rate related to changing running speed. The internal model parameters like delays (DS and DR, Figure 1) are adjusted to optimal values, in which simulated and original heart rates correspond best.

After the individual calibration of the model to the athlete, the model can be used for simulations. The user only has to put in a speed progress and gets out the corresponding heart rate progress for the adapted athlete. In addition, the simulation provides the reserve value, which provides an indication of nearness to the overloading mechanism:

$$
\text { Reserve }[\mathrm{t}]=1-\mathrm{SP}[\mathrm{t}]
$$

To avoid overloading, this value shouldn't be zero or negative. We utilize this value for the simulation of AT. For constant running speed, HR converges to an individual level and increments slightly at most. If running speed exceeds a critical level, HR would increase excessively. In our model, this would be the moment where overloading mechanism begins and reserve value would become negative.

The determination method of the simulated AT by PerPot simulates several 60 minute constant endurance runs. The speed increases minimally in every simulation run as long as the reserve doesn't reach zero during this simulation. If the reserve (7) is greater than zero over the whole simulation, the athlete wouldn't reach his limit during the simulation with the speed used. Once the reserve gets zero, the athlete reaches his limit and we define the simulated AT as the simulated heart rate at the end of this simulation.

\subsection{Statistics}

Statistical analysis of the heart rate values at the AT were performed by using SPSS Statistics 21 by IBM. Initially, thresholds of all five models were checked on normal distribution by means of the Shaprio-Wilk test. Homogeneity of variances were checked by means of the Levene test. Both tests are required for further analysis with correlation coefficients.

The correlation between the simulated PerPot ATs and the lactate based ATs was evidenced with the Pearson correlation value (r). As measurement for the absolute 
consistency the intra class correlation coefficient (ICC) was determined.

Bland-Altman plots were used to visualise the variation of differences between simulated and lactate based thresholds [23]. This graphical means is useful to assess whether the two series, each having some errors, are comparable.

\section{Results}

Analysis of the four lactate-based thresholds and the PerPot threshold show similar mean HR over all participants (PerPot: 177.62 \pm 8.55 ; Dickhuth: 174.69 \pm 9.11 ; OBLA 3.5: 175.54 \pm 8.56 ; Keul: 175.46 \pm 9.60 ; Simon: 173.62 \pm 9.19 ).

Results of each threshold concept were checked positively on normal distribution and homogeneity of variance by Shaprio-Wilk test and Levene test, respectively.
All Pearson correlation coefficients between the different thresholds showed positive correlations with high significance at the 0.001 level (two-tailed), as shown in table 2. Overall lowest and highest correlation were calculated between lactate based ATs (OBLA 3.5 - Simon $\mathrm{r}=0.880$ and Simon - Dickhuth $\mathrm{r}=0.994)$. The correlation values between PerPot AT and lactate based ATs were between $r=0.883$ and $\mathrm{r}=0.895$.

ICC values show very high correlation coefficients between the different thresholds (see table 2), except the value between PerPot and Simon ( $\mathrm{ICC}=0.894)$. Overall highest correlation was again between Simon and Dickhuth (ICC $=0.994$ ). The ICC between PerPot AT and all lactate based ATs show the validity of the computer science model based AT with coefficients between ICC $=0.894$ and ICC $=0.932$.

Table 2. Intraclass correlation coefficient (ICC), Pearson correlation ( $\mathrm{r}$ ) and significance between the different thresholds $(\mathrm{N}=13)$

\begin{tabular}{|c|c|c|c|c|c|c|}
\hline & & Perpot & OBLA 3.5 & Keul & Simon & Dickhuth \\
\hline \multirow{3}{*}{ PerPot } & ICC & \multirow{3}{*}{-} & $.932^{* *}$ & $.925^{* *}$ & $.894^{*}$ & $.916^{* *}$ \\
\hline & $\mathrm{r}$ & & $.892^{+}$ & $.883^{+}$ & $.895^{+}$ & $.889^{+}$ \\
\hline & $\begin{array}{l}\text { Significance } \\
\text { (two-tailed) }\end{array}$ & & .000 & .000 & .000 & .000 \\
\hline \multirow{3}{*}{ OBLA 3.5} & ICC & & \multirow{3}{*}{ - } & $.941^{* *}$ & $.928^{* *}$ & $.936^{* *}$ \\
\hline & $\mathrm{r}$ & & & $.885^{+}$ & $.880^{+}$ & $.876^{+}$ \\
\hline & $\begin{array}{l}\text { Significance } \\
\text { (two-tailed) }\end{array}$ & & & .000 & .000 & .000 \\
\hline \multirow{3}{*}{ Keul } & ICC & & & \multirow{3}{*}{ - } & $.978^{* *}$ & $.992^{* *}$ \\
\hline & $\mathrm{r}$ & & & & $.976^{+}$ & $.987^{+}$ \\
\hline & $\begin{array}{l}\text { Significance } \\
\text { (two-tailed) }\end{array}$ & & & & .000 & .000 \\
\hline \multirow{3}{*}{ Simon } & ICC & & & & \multirow{3}{*}{ - } & $.994^{* *}$ \\
\hline & $\mathrm{r}$ & & & & & $.994^{+}$ \\
\hline & $\begin{array}{l}\text { Significance } \\
\text { (two-tailed) }\end{array}$ & & & & & .000 \\
\hline \multirow{3}{*}{ Dickhuth } & ICC & & & & & \multirow{3}{*}{ - } \\
\hline & $\mathrm{r}$ & & & & & \\
\hline & $\begin{array}{l}\text { Significance } \\
\text { (two-tailed) }\end{array}$ & & & & & \\
\hline
\end{tabular}

Note. *. High correlation $0.7<\mathrm{r} \leq 0.9$;

**. Very high correlation $r>0.9+$. Correlation is significant at the 0.001 level (two-tailed)
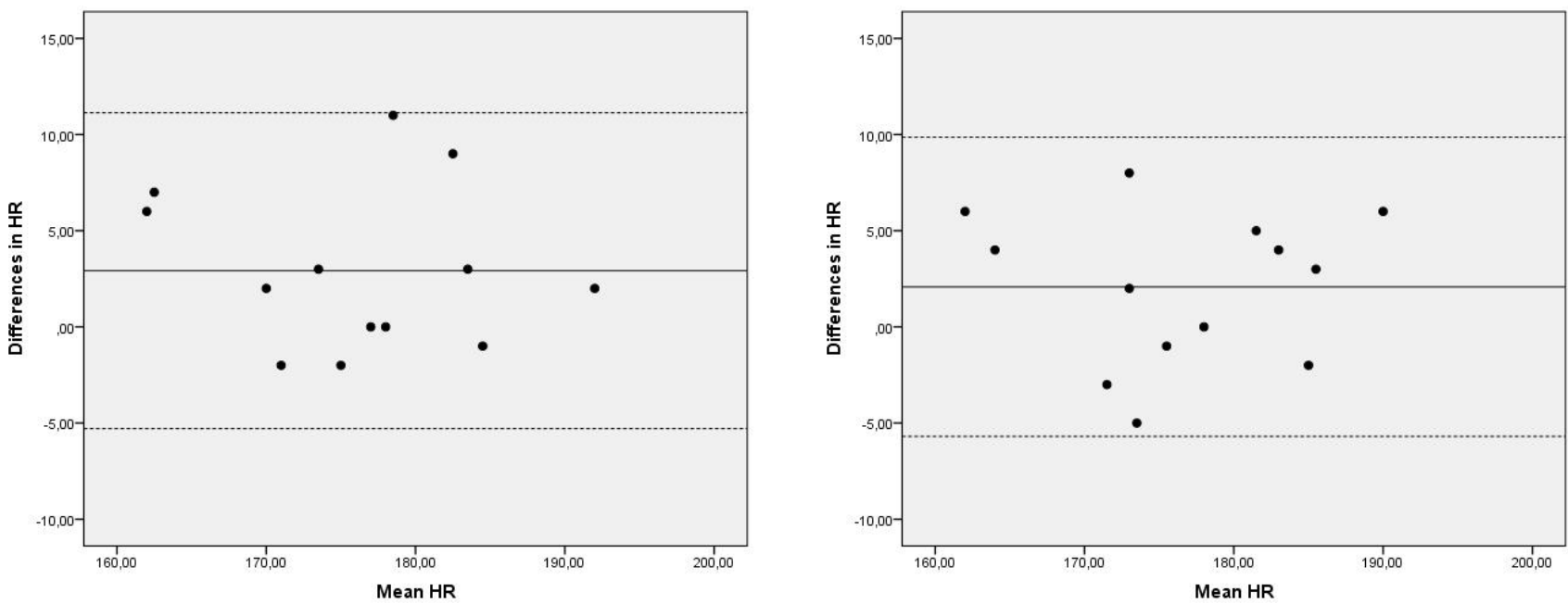

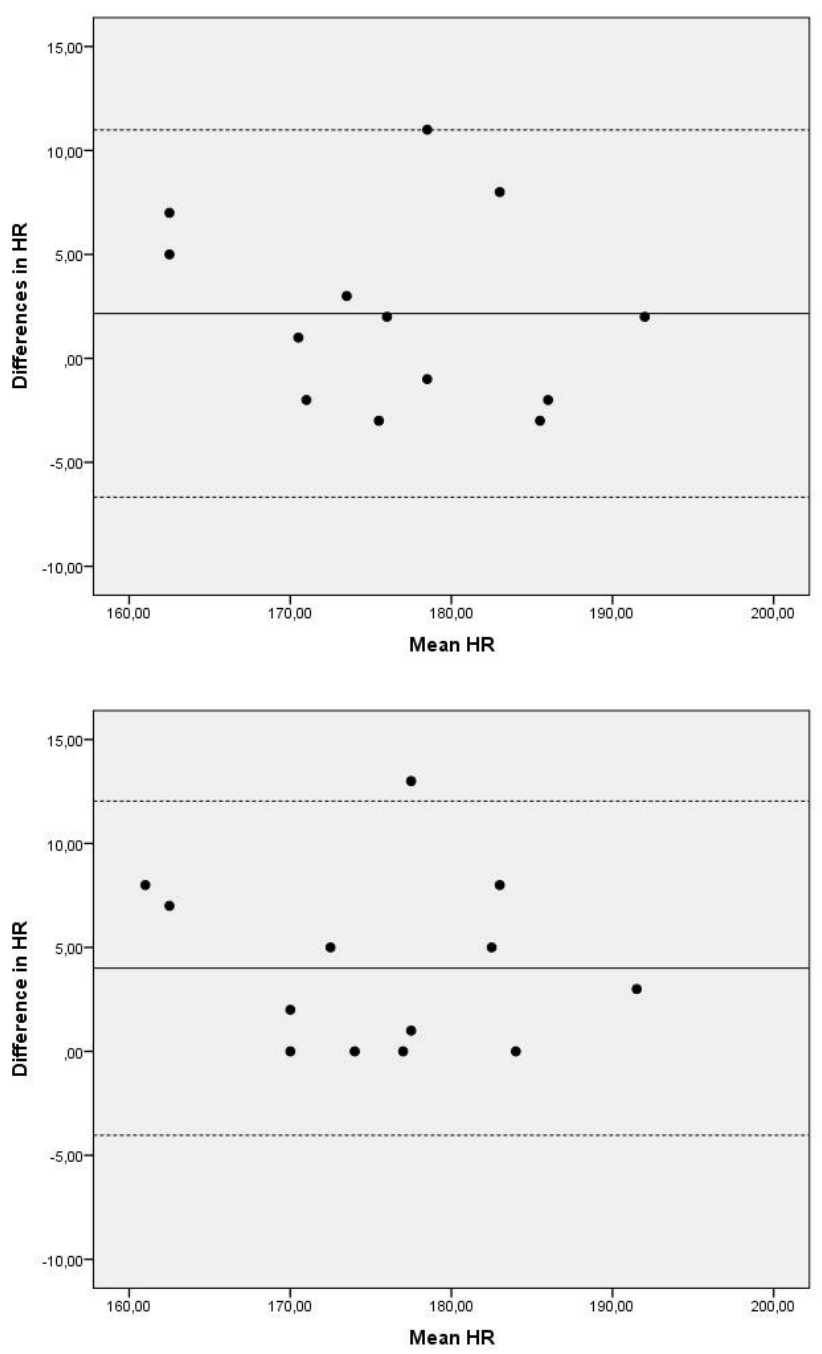

Figure 2. Four Bland-Altman plots comparing PerPot simulated heart rates (HR) at anaerobic thresholds (AT) and four different HRs at lactate based ATs a) Dickhuth; b) OBLA 3.5; c) Keul; d) Simon

Figure 2 shows the differences in heart rate values between the lactate based ATs and PerPot AT in Bland-Altman plots.

\section{Discussion}

The main finding of the present study is a high comparability of conventional lactate based thresholds and the new non-invasive simulated threshold using a computer science model named PerPot.

Today, prescribing exercise intensities by heart rate zones determined related to AT's is the gold-standard [2,3]. However, there are many points of criticism in conjunction with AT's. For example, Mann et al. [3] criticize the failure of verifying threshold measurements, which may create individual variation in blood lactate accumulation. This leads to a practical advantage of the measurement of VO2max and HRmax over threshold measurements, although they should not be used from the theoretical aspect. Another disadvantage is the rarely reported validation of
AT's by the MLSS [7], which can be determined reliably and with low day-to-day variability [24]. Hauser et al. [25] compared Dickhuth's threshold with MLSS in cycling. However, they compared the power instead of the heart rate. They found a significant underestimation of Dickhuth's threshold compared to MLSS (-37.4 $\pm 26.4 \mathrm{~W})$. Furthermore, their Bland-Altman plots showed noticeably large spreads of differences between MLSS and their examined thresholds. In contrast, PerPot thresholds tend to be higher than Dickhuth's thresholds (Figure 2a). This could mean that the simulated AT by PerPot leads to an even better MLSS approximation, but that has to be shown in future investigations. Missing further validation might be due to the difficulty of determining MLSS by several constant endurance loads. Nevertheless, AT determination using lactate is the gold-standard and therefore has to be used as the first validation value.

The only meaningful attempt to approximate AT by a non-invasive method was introduced by Conconi [10]. Carey et al. [26] in fact validated the Conconi threshold by means of a ventilatory AT, but ill-advised it for practicality because of relatively large total errors. It is even more limited because heart rate deflection points cannot be found in some cases [11].

The first sports computer science method tried to determine the AT using a multi-layer perceptron [12]. Erdogan et al. achieved a correlation $\mathrm{r}=0.766$ between their method and OBLA [15], which is lower than PerPot correlation $(\mathrm{r}=0.892)$. The disadvantage of this method is the dependency of the used training set for the neural network. In this case the data of young soccer players were used, so ATs can only be estimated for other young soccer players. The method cannot be used generally, because it doesn't consider the physiology of the athletes, but uses statistical values such as age, body mass and height instead.

In contrast, our non-invasive approach models the individual delayed reaction of heart rate to changing running speed. It requires a maximal graded test of an athlete, used as well in sport medical performance diagnostic, to determine individual delays of heart rate changes caused by speed changes. Based on these delays and since PerPot has an overload mechanism, the AT can be simulated by several constant endurance runs.

The present study shows a high comparability of this new method compared to the conventional lactate-based thresholds. Compared to the most applied AT determination by Dickhuth [7, 14], simulated PerPot AT shows high correlations with $\mathrm{r}=0.889$ and $\mathrm{ICC}=0.916$. These positive results implicate a useful method to keep AT up-to-date by periodical determination. Regarding the population, these results are limited to handball players of medium performance level. Further studies must show up transferability of significance to other populations regarding e.g. other sports or performance levels. However, our method models heart rate reaction to load in general. Therefore, it is most likely transferable with similar 
significantly high correlations to basic population and not limited to any special population.

One advantage of the PerPot method is the low cost. An athlete only needs a heart rate monitor, which has the possibility to record data and an optional speed sensor. Thus, popular sports athletes, which normally does not have the opportunity of an expensive sports medicine diagnostic, could make use of this individual training optimization as well. Another advantage is the easy portability of results to field, because the graded incremental test without lactate extraction can be performed everywhere and hence is not tied to a laboratory. The portability to field is important due to limitations of comparing laboratory and field lactate tests. Kunduracioglu et al. [27] found significant differences in HR and blood lactate responses between treadmill and field running. This is due to other conditions in field, e.g. temperature, air resistance and so on. Thus, a field test should be preferred, because training and competition is done in field, normally.

\section{Conclusions}

In this study we established a new method to determine the AT by means of a computer science model named PerPot. Statistical analysis showed high correlations between this method and conventional lactate-based methods. The PerPot AT should be validated further by a larger dataset and even now compared directly to MLSS. Nevertheless, PerPot brings a lot of advantage in exercise prescription.

\section{Practical Implications}

- Because of adaption of physiological parameters to exercise, AT and the corresponding arrangement of intensities change

- If AT is used for exercise prescription, it should be determined periodically after a certain time, otherwise an athlete may exercise in wrong intensities after a certain time

- PerPot offers a possibility of keeping AT up-to-date by periodical determination, without further costs

- Amateur athletes that use HFmax or other possibilities for exercise prescription have the chance to optimize their training using a more individual parameter

- It should be noted, that PerPot does not claim to replace sports medicine diagnostic, which provide more than just determining AT

\section{REFERENCES}

[1] D. J. Smith. A framework for understanding the training process leading to elite performance, Sports Medicine, Vol. 33, No.15, 1103-1126, 2003.
[2] P. Hofmann, G. Tschakert. Special needs to prescribe exercise intensity for scientific studies, Cardiology research and practice, 2011.

[3] T. Mann, R. P. Lamberts, M. I. Lambert. Methods of Prescribing Relative Exercise Intensity: Physiological and Practical Considerations, Sports Medicine, Vol.43, No.7, 613-625, 2013.

[4] F. Scharhag-Rosenberger, T. Meyer, N. Gaessler, O. Faude, W. Kindermann. Exercise at given percentages of VO2max: Heterogeneous metabolic responses between individuals, Journal of Science and Medicine in Sport, Vol.13, No.1, 74-79, 2010 .

[5] F. A. da Cunha, P. d. T. Veras Farinatti, A. W. Midgley. Methodological and practical application issues in exercise prescription using the heart rate reserve and oxygen uptake reserve methods, Journal of Science and Medicine in Sport, Vol.14, No.1, 46-57, 2011.

[6] G. D. Wells, H. Selvadurai, I. Tein. Bioenergetic provision of energy for muscular activity, Paediatric Respiratory Reviews, Vol.10, No.3, 83-90, 2009.

[7] O. Faude, W. Kindermann, T. Meyer. Lactate Threshold Concepts How Valid are They?, Sports Medicine, Vol.39, No.6, 469-490, 2009.

[8] K. Roecker. Lactate Diagnostics in Sports Medicine: Technical Conditions and Fields of Application, Deutsche Zeitschrift Für Sportmedizin, Vol.64, No.12, 367-371, 2013.

[9] P. Foxdal, B. Sjodin, A. Sjodin, B. Ostmann. Validity and accuracy of blood lactate measurement for prediction of maximal endurance running capacity - dependency of analyzed blood media in combination with different designs of the exercise test, International Journal of Sports Medicine, Vol.15, No.2, 89-95, 1994.

[10] F. Conconi, M. Ferrari, P. G. Ziglio, P. Droghetti, L. Codeca. Determination of the anaerobic threshold by a non-invasive field-test in runners, Journal of Applied Physiology, Vol.52, No.4, 869-873, 1982.

[11] P. Hofmann, R. Pokan, K. Preidler, H. Leitner, D. Szolar, B. Eber, G. Schwaberger. Relationship between heart-rate threshold, lactate turn point and myocardial-function, International Journal of Sports Medicine, Vol.15, No.5, 232-237, 1994.

[12] A. Erdogan, C. Cetin, H. Goksu, R. Guner, M. L. Baydar. Non-invasive detection of the anaerobic threshold by a neural network model of the heart rate-work rate relationship, Proceedings of the Institution of Mechanical Engineers Part P-Journal of Sports Engineering and Technology, Vol.223, No.3, 109-115, 2009.

[13] F. Faul, E. Erdfelder, A. Buchner, A.-G. Lang. Statistical power analyses using $\mathrm{G}^{*}$ Power 3.1: Tests for correlation and regression analyses. Behavior Research Methods, Vol.41, 1149-1160, 2009.

[14] H.-H. Dickhuth, L. Yin, A. Niess, K. Rocker, F. Mayer, H. C. Heitkamp, T. Horstmann. Ventilatory, lactate-derived and catecholamine thresholds during incremental treadmill running: Relationship and reproducibility, International Journal of Sports Medicine, Vol.20, No.2, 122-127, 1999.

[15] A. Mader, H. Liesen, H. Heck. Evaluation of the sport specific 
endurance performance capacity in laboratory, Sportarzt Sportmedizin, Vol.27, 80-88, 109-112, 1976.

[16] J. Keul, G. Simon, A. Berg, H.-H. Dickhuth, I. Goerttler, R. Kübel. Determination of the individual anaerobic threshold for performance assessment and training prescribtion, Deutsche Zeitschrift für Sportmedizin, Vol.30, 212-218, 1979.

[17] G. Simon, A. Berg, H.-H. Dickhuth, A. Simon-Alt, J. Keul. Determination of the anaerobic threshold dependent on age and performance assessment, Deutsche Zeitschrift für Sportmedizin, Vol32, 7-14, 1981

[18] H. Heck, G. Hess, A. Mader. Comparative investigation on different lactate based thresholds, Deutsche Zeitschrift für Sportmedizin, Vol.36, No.1, 19-25, 1985.

[19] Polar Electro, Polar HRM2 File Format Description, Online available from http://www.polar.com/files/Polar_HRM_file format.pdf.

[20] S. Endler. Adaptation of the meta model PerPot to endurance running for optimization of training and competition, Doctoral dissertation, Johannes Gutenberg University Mainz, 2013.

[21] J. Perl. PerPot - a meta-model and software tool for analysis and optimisation of load-performance-interaction, International Journal of Performance Analysis of Sport, Vol.4 No.2, 61-73, 2004.
[22] J. D. Brooke, E. J. Hamley. The heart-rate - physical work curve analysis for the prediction of exhausting work ability, Medicine and Science in Sports, Vol.4, No1, 23-26, 1972.

[23] J. M. Bland, D. G. Altman. Statistical methods for assessing agreement between two methods of clinical measurement, International Journal of Nursing Studies, Vol.47, No.8, 931-936, 2010.

[24] T. Hauser, D. Bartsch, L. Baumgärtel, H. Schulz. Reliability of Maximal Lactate-Steady-State, International Journal of Sports Medicine, Vol.34, No.3, 196-199, 2013.

[25] T. Hauser, J. Adam, H. Schulz. Comparison of Selected Lactate Threshold Parameters with Maximal Lactate Steady State in Cycling, International Journal of Sports Medicine, Vol.35, No.6, 517-521, 2014.

[26] D. G. Carey, G. J. Pliego, R. L. Raymond. A comparison of different heart rate deflection methods to predict the anaerobic threshold, European Journal of Sport Science, Vol.8, No.5, 315-323, 2008.

[27] B. Kunduracioglu, R. Guner, B. Ulkar, A. Erdogan. Can heart rate values obtained from laboratory and field lactate tests be used interchangeably to prescribe exercise intensity for soccer players?, Advances in Therapy, Vol.24, No.4, 890-902, 2007. 